\title{
UTILIZAÇÃO DE BIOSSURFACTANTE COMO SUBSTITUTO AOS EMULSIFICANTES SINTÉTICOS EM MAIONESES
}

\author{
J.M.CAMPOS ${ }^{1}$, T.L.M. STAMFORD ${ }^{2}$ e L.A.SARUBBO ${ }^{3}$ \\ ${ }^{1}$ Universidade Federal de Pernambuco, Departamento de Engenharia Química \\ ${ }^{2}$ Universidade Federal de Pernambuco, Departamento de Nutrição \\ ${ }^{3}$ Universidade Católica de Pernambuco, Centro de Ciência e Tecnologia \\ E-mail para contato: jenyffermcampos@gmail.com
}

\begin{abstract}
RESUMO - Tem sido cada vez mais crescente o uso de biossurfactantes na indústria de alimentos. Devido a isto, o biossurfactante produzido por Candida utilis foi avaliado como agente emulsificante em maioneses. Seis formulações foram determinadas utilizando goma guar, carboximetilcelulose e o biossurfactante, isolado ou combinados entre si, avaliando-se a estabilidade das maioneses semanalmente mantidas sob refrigeração a $4^{\circ} \mathrm{C}$ por trinta dias. Ao final do experimento, observou-se que apenas a formulação contendo goma guar e o biossurfactante mantiveram a emulsão estável ficando as demais formulações com a segregação de duas fases, a aquosa e a lipídica. Com isto, pode-se concluir que o biossurfactante isolado ou apenas os estabilizantes goma guar e carboximetilcelulose não possuem a capacidade de manter a formulação estável por trinta dias e que o biossurfactante apresenta potencial emulsificante desde que seja utilizado combinado com o estabilizando goma guar.
\end{abstract}

\section{INTRODUÇÃO}

Devido suas propriedades físico-químicas, o uso de biossurfactantes fazem deles um grupo atrativo de compostos com uso potencial em uma variedade de aplicações industriais e biotecnológicas, como aditivos em alimentos, cosméticos e detergentes (Pacwa-Plocinizak et al., 2011; Marchant e Banat, 2012). O potencial uso de biossurfactantes na indústria de alimentos são conferidas às propriedades emulsificantes, espumantes, umectantes, solubilizantes e agentes antiadesivos (Muthusamy et al., 2008). A propriedade mais comum, na indústria alimentícia é a habilidade de formar emulsões estáveis que promovem textura e cremosidade em produtos lácteos. Biossurfactantes são também usados para retardar aglomeração, solubilizar óleos aromáticos e promover propriedades organolépticas em produtos de panificação e formulações com sorvete e estabilizar a gordura durante a fritura (Banat et al., 2010; Nitschke e Costa, 2007).

É descrito na literatura que em 2004 aproximadamente $\pm 0,2$ milhões de toneladas de surfactantes foram usados em alimentos em um total anual de produção de aproximadamente 10 milhões de toneladas em 2007 (Van Bogaert et al., 2007). Entre as espécies de microrganismos produtores de biossurfactante, o gênero Candida, e muitas espécies tem sido sugeridas como 
potencial uso benéfico em alimentos incluindo $C$. intermedia, C. maltosa, S. versatilis e $C$. zeylanoides (Bourdichon et al., 2012).

Na natureza, surfactantes naturais como a lecitina da gema do ovo e várias proteínas do leite são usadas no preparo de muitos produtos alimentícios como maionese, molhos para salada, sobremesas, etc. Mais recentemente, surfactantes sintéticos como ésteres de sorbitano e seus etoxilados e ésteres de sacarose tem sido usado em emulsões alimentícias. Recentemente, Campos et al., (2013) publicaram uma revisão onde descrevem a aplicação dos biossurfactantes na indústrias de alimentos devido suas propriedades emulsificantes, desempenhando um papel na consistência e textura, solubilização de aromas e como estabilizante de sistemas aerados, favorecendo assim o uso em indústrias alimentícias diversificadas.

Desta forma, este estudo objetivou o uso do biossurfactante produzido por Candida utilis com propriedade emulsificante, isolado ou combinado com estabilizantes, na produção de uma emulsão estável.

\section{MATERIAL E MÉTODOS}

\subsection{Material}

Microrganismo: Candida utilis (UFPEDA 1009) foi utilizada como produtora de agente surfactante procedente do Departamento de Antibióticos da Universidade Federal de Pernambuco. As culturas foram mantidas a $5^{\circ} \mathrm{C}$ em meio Yeast Mold Agar (YMA) e o crescimento do inóculo foi realizado em meio YMB (Yeast Mold Broth), o qual possui a mesma composição do meio YMA, excluindo-se o ágar. Repiques foram mensalmente realizados para manter a viabilidade celular.

Meio de produção e substratos: Para a produção do biossurfactante utilizou-se meio mineral contendo: $0,2 \%$ de $\mathrm{NH}_{4} \mathrm{NO}_{3}, 0,01 \%$ de $\mathrm{KH}_{2} \mathrm{PO}_{4}, 0,5 \%$ de $\mathrm{MgSO}_{4} .7 \mathrm{H}_{2} \mathrm{O}, 0,01 \%$ de $\mathrm{FeCl}_{3}, 0,01 \%$ de $\mathrm{NaCl}, 0,1 \%$ de extrato de levedura, $6 \%$ de glicose acrescido de $6 \%$ de óleo de fritura de canola. Os ingredientes foram dissolvidos e o meio esterilizado em autoclave a $121^{\circ} \mathrm{C}$ por 20 minutos, sendo o pH final de 5,7.

\subsection{Métodos}

Preparação do inóculo: O inóculo foi padronizado transferindo-se a levedura para um tubo contendo o meio YMA, a fim de se obter uma cultura jovem. Em seguida, a amostra foi 
transferida para frascos contendo $50 \mathrm{~mL}$ do meio YMB, sendo então incubados sob agitação de $200 \mathrm{rpm}$ a $28^{\circ} \mathrm{C}$ durante 24 horas. Após este período, foram realizadas diluições até obtenção da concentração final de células desejada $\left(10^{8}\right.$ células $\left./ \mathrm{mL}\right)$.

Produção do biossurfactante: As fermentações para produção dos biossurfactantes com atividade de emulsificação foram realizadas em frascos de Erlenmeyer de $500 \mathrm{~mL}$ contendo 100 $\mathrm{mL}$ do meio de produção. Os frascos foram incubados com a suspensão celular e mantidos sob agitação orbital de $200 \mathrm{rpm}$ durante 144 horas, à temperatura de $28^{\circ} \mathrm{C}$. Após o cultivo, alíquotas foram retiradas para determinação da tensão superficial, da atividade de emulsificação e do rendimento em produto isolado.

Isolamento do biossurfactante: 0 líquido metabólico foi inicialmente filtrado em membrana millipore de $0,45 \mu \mathrm{m}$ para retirada das células e então submetido aos processos de extração do líquido metabólico pelo método de Cameron et al. (1988).

Aplicação do biossurfactante como substituto de emulsificantes: A propriedade emulsificante do biossurfactante foi testada na formulação de um molho para uso em saladas, com a seguinte composição: $40 \%$ de óleo de girassol (Bunge), 40,3\% de água, 10\% de vinagre (comercial), 4\% de ovo em pó (Naturovos LTDA, Brasil), 2\% de açúcar, 2\% de sal, 1\% de farinha de mostarda (todos comerciais) e 0,5\% de amido instantâneo (Unilever LTDA, Brasil). O biossurfactante isolado de Candida foi usado na concentração de $0.7 \%$ combinado adicionalmente com o acréscimo de $0.2 \%$ de goma guar e $0.2 \%$ carboximetilcelulose (CMC) (Cromato Produtos Químicos LTDA, Brasil), na formulação de seis diferentes maioneses descritas na tabela 1. Os ingredientes foram misturados com o auxílio de um agitador durante um minuto à temperatura ambiente. Os molhos obtidos foram armazenados a $4^{\circ} \mathrm{C}$ durante um mês para inspeção visual de sua aparência (Torabizadeh et al., 1996) e avaliados semanalmente quanto à estabilidade da emulsão.

Tabela 1 - Ingredientes adicionais utilizados na formulação de maionese

\begin{tabular}{|c|c|}
\hline Formulação & Ingredientes testados \\
\hline 1 & Carboximetilcelulose + goma guar \\
\hline 2 & Carboximetilcelulose \\
\hline 3 & Carboximetilcelulose + biossurfactante isolado de Candida \\
\hline 4 & Goma guar \\
\hline 5 & Goma guar + biossurfactante isolado de Candida \\
\hline 6 & Biossurfactante isolado de Candida \\
\hline
\end{tabular}

\section{RESULTADOS E DISCUSSÃO}

A figura 1 apresenta as maioneses formuladas e seu comportamento ao longo das quatro semanas de avaliação. Logo na segunda semana, a formulação seis apresentou separação aquosa 
na base do recipiente e que, ao final do prazo estabelecido, tornou-se mais evidente. Pode-se visualizar também que todas as formulações, exceto a 5, desestabilizaram apresentando acúmulo de líquido, evidenciando que não foram $100 \%$ estáveis. Ao abrir todos os recipientes, no final do experimento, percebeu-se uma consistência fluida nas formulações 1, 2 e 3, apresentando a formulação 5 uma consistência cremosa, uniforme e estável.

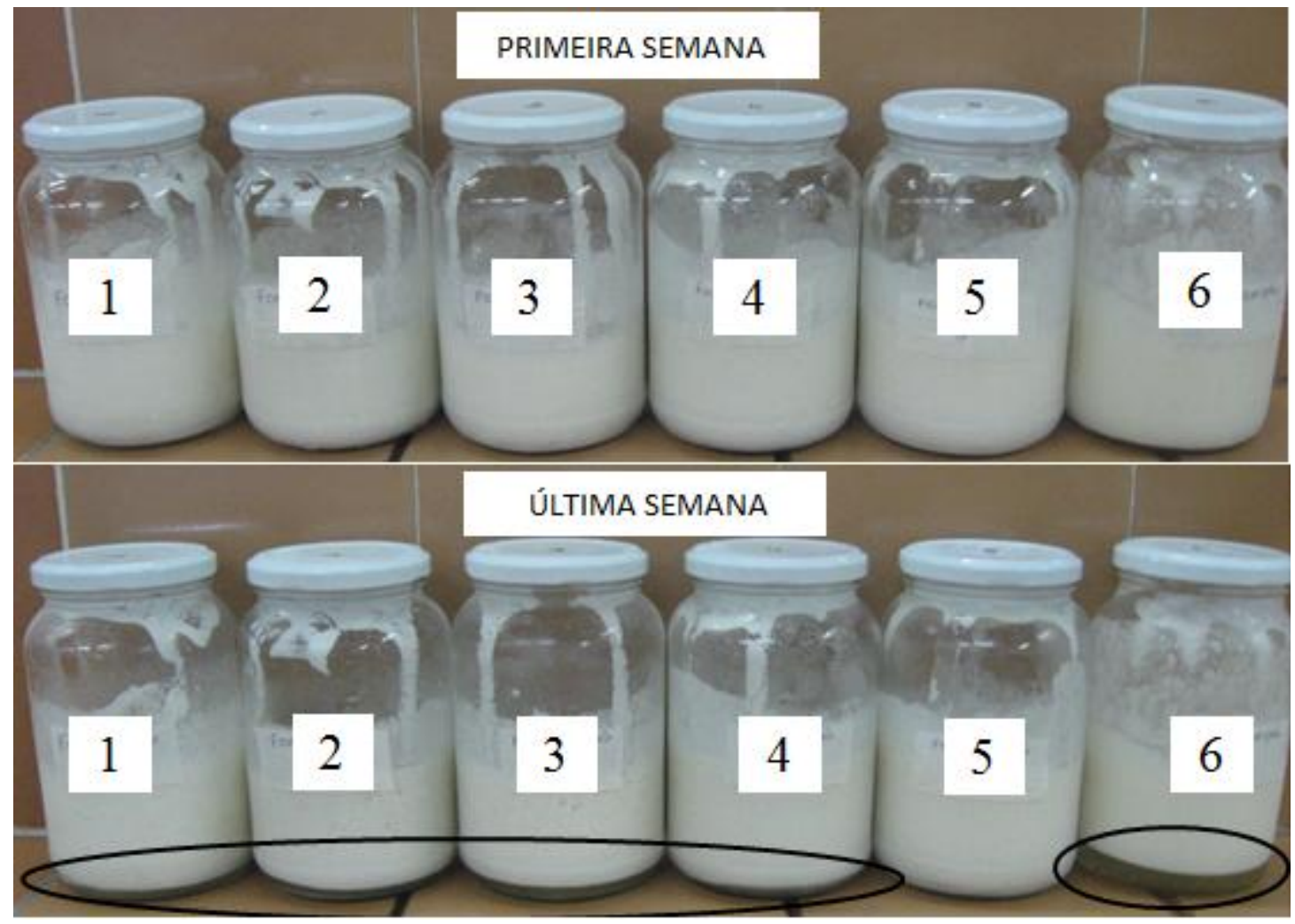

Figura 1- Comportamento das maioneses formuladas com CMC e biossurfactante a $0.7 \%$ frente à estabilidade da emulsão formada durante quatro semanas sob refrigeração.

Segundo a ANVISA (2005), maionese é o produto cremoso em forma de emulsão estável, óleo em água, preparado a partir de óleo(s) vegetal(is), água e ovos podendo ser adicionado de outros ingredientes desde que não descaracterizem o produto. É proibida a adição de corantes, e a porcentagem máxima de amido permitida em sua composição é de $0,5 \%$. As emulsões são colóides, misturas heterogêneas compostas de partículas minúsculas suspensas em outro material imiscível.

Um agente emulsionante/emulsificante é a substância que torna possível a formação ou manutenção de uma mistura uniforme de duas ou mais fases imiscíveis no alimento (Portaria 540/97), ou seja, são agentes anfipáticos que alteram as propriedades de alguns alimentos para que eles possam ser misturados, formando uma emulsão. $\mathrm{O}$ emulsificante possui moléculas com 


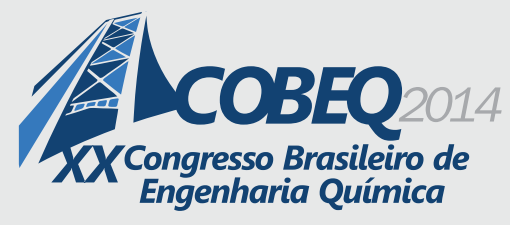

19 a 22 de outubro de 2014
Florianópolis/SC

extremidade polar, que é atraída pela água, e outra extremidade apolar, que é atraída pelo óleo. Neste estudo, por definição, o biossurfactante atuaria como agente emulsificante devido apresentar tal propriedade. Biossurfactantes podem estabilizar (emulsificar) ou desestabilizar (deemulsificar) a emulsão (Muthusamy et al., 2008).

A portaria $\mathrm{n}^{\circ} 372$ (BRASIL, 1999), considerando que o uso dos aditivos deve ser limitado a alimentos específicos, em condições específicas e no menor nível para alcançar o efeito desejado (Portaria SVS/MS n. ${ }^{\circ}$ 540/97) aprovou o uso de aditivos com a função estabilizante para o produto maionese, configurando dentre eles a goma guar e a carboximetilcelulose.

Dentre os óleos mais utilizados na elaboração de maionese, estão o óleo de soja e o óleo de girassol. Alternativamente, outros tipos de óleos estão sendo utilizado na elaboração de maioneses, como o óleo de palma (Chandrasekaran e Bahkali, 2013). A gema do ovo é uma emulsão natural do óleo em água em combinação com proteínas, lecitinas e outros fosfolipídios. A gema, como uma emulsão, forma o núcleo em que a emulsão da maionese é feita, afetando a viscosidade e força da emulsão final.

Em relação aos demais ingredientes descritos na formulação da maionese está a farinha de mostarda. As sementes da mostarda branca ou amarela (Brassica alba) contém compostos especiais como glucosinolatos. Estes compostos caracterizam este sabor de mostarda e produtos com mostarda (Tsao et al., 2000; Nadarajah et al., 2005). Apresenta diversas características funcionais, entre elas emulsificante, estabilizante, aglutinantes, conservante e antioxidante. Como agente emulsificante, em produtos tais como maioneses e molhos para saladas, a mostarda em pó é utilizada em níveis de 0,2-0,4\% do total como emulsificante adicional e também estabilizante, além de conferir aroma ao produto. Como estabilizante, as finas partículas da mostarda se acumulam na interface óleo/água agindo assim como uma proteção física contra uma quebra da emulsão.

Além de conferir propriedade antimicrobiana, amido e outros hidrocolóides ou gomas são usados mais frequentemente como texturizantes na indústria alimentícia sendo utilizadas em emulsões para estabilizá-las. Tanto a goma guar e carboximetilcelulose, que foram testadas nas formulações a $0.2 \%$, foram utilizadas como estabilizantes em maionese e podem conferir estas propriedades. Em relação à isto, percebe-se na figura 1, que a manutenção da emulsão mais estável, composta com goma guar e biossurfactante, foi conferido pelo poder estabilizante da goma guar e emulsificante do biossurfactante. Isto pode ser comprovado ao se analisar a formulação 6, a qual não apresentou adição de nenhum agente estabilizante havendo a segregação de duas fases visivelmente distintas. Em emulsões relativamente estáveis também houve separação de uma camada, no caso de óleo, em emulsões de alginato após 30 dias de avaliação (Sato et al 2014). Já a levedura de cerveja $\beta$-glucano estabilizou a emulsão por 91 dias a $4-6^{\circ} \mathrm{C}$ e pode ser usada como substituto de gordura em maionese (Marinescu et al., 2011).

A inulina testada entre $0-10 \%(\mathrm{p} / \mathrm{v})$, por Alimi et al. (2013), obteve o melhor resultado na concentração de $5 \%(\mathrm{p} / \mathrm{v})$. O uso de $0-1 \%(\mathrm{p} / \mathrm{v})$ goma de alfarroba fornece importante informação sobre a influência de hidrocolóides nas propriedades de modelos multicomponentes em emulsões (Chung et al., 2014). O efeito da goma guar e xantana também pode afetar as 


\section{9 a 22 de outubro de 2014 \\ Florianópolis/SC}

propriedades reológicas do amido, também utilizado na formulação, induzindo efeitos indiretos para melhor preservação da estrutura granular (Heyman et al., 2014). Paraskevopoulou et al. (2007) mostrou que polissacarídeos (goma arábica e alginate de propileno glicol) tiveram habilidade de induzir aumento de viscosidade. A aplicação de gel konjac em escala micrométrica indicou que gorduras em maionese substituídas por gel konaj, não mais que $30 \%$, foi bem aceito e demostrou bom potencial para ser usado como análogo de maionese (Li et al., 2014).

A maioria dos complexos colóides e emulsões que são usados em alimentos e produtos alimentícios são difíceis de estabilizar por causa da grande número de microestruturas de combinações de proteínas, carboidratos e lipídios presentes. Este número infinito de combinações são organizados e arranjados em complexos internos de microestruturas com vários tipos de arranjos entre dispersões, emulsões, espumas, géis, etc. (Kralova e Slöblom, 2009). Como dito anteriormente, a adição do biossurfactante isolado (formulação 6) não foi eficaz na manutenção da emulsão por 30 dias. Soforolipídios de T. bombicolatem tem se mostrado reduzir a tensão superficial e interfacial mas não bons emusificantes (Cooper e Cavalero, 2003). Em contraste, liposan não reduz bem a tensão superficial mas tem sido usado com sucesso na emulsificação de de óleos comestíveis (Cirigliano e Carman, 1985).

\section{CONCLUSÃO}

Poucos trabalhos na literatura relatam, de fato, a aplicação de biossurfactante na formulação de novos produtos alimentícios inferindo apenas o provável uso em alimentos, diferente do presente trabalho. Com isto, pode-se concluir que a formulação contendo goma guar e biossurfactante isolado de Candida associado obteve melhor estabilidade após quatro semanas de avaliação, comprovando a função emulsificante do biossurfactante associado com a função estabilizante da goma guar. Desta forma, este bioemulsificante pode ser utilizado na indústria alimentícia conferindo tal propriedade em emulsões.

\section{REFERÊNCIAS}

ALIMI, M.; MIZANI, M.; NADERI, G.; MORTAZAVIAN, A.M.; MOGHADAM, M.B. Development of low-fat mayonnaise containing combined mixtures of different types of inulin. J. Food, Agric. Environ., v. 11, p. 99-104, 2013.

BANAT, I.M.; FRANZETTI, A.; GANDOLFI, I.; BESTETTI, G.; MARTINOTTI, M.G.; FRACCHIA, L.; SMYTH, T.J.; MARCHANT, R. Microbial biosurfactants production, applications and future potential. Appl. Microbiol. Biotechnol., 87, 427-444, 2010.

BRASIL, Portaria no 372 - Agência Nacional de Vigilância Sanitária - Ministério da Saúde, de 
26 de abril de 1999. http://www.anvisa.gov.br/legis/portarias/372_99.htm

BRASIL, Portaria n 540 - Agência Nacional de Vigilância Sanitária - Ministério da Saúde, de 27 de outubro de 1997.

BRASIL, Resolução RDC no 276 - Agência Nacional de Vigilância Sanitária - Ministério da Saúde, de 26 de setembro de 2005.

CAMERON, D.R.; COOPER, D.G.; NEUFELD, R.J. The mannoprotein of Saccharomyces cerevisiae is an effective bioemulsifier. Appl. Environ. Microbiol., v. 54, p. 1420-1425, 1988.

CAMPOS, J. M.; STAMFORD; T. L. M.; RUFINO, R. D.; LUNA, J. M.; BANAT, I. M.; SARUBBO, L. A. Microbial biosurfactants as additives for food industries. Biotechnol. Progress, v. 29, p. 1097 - 1108, 2013.

CHANDRASEKARAN, M.; ALI H. BAHKALI. Valorization of date palm (Phoenix dactylifera) fruit processing by-products and wastes using bioprocess technology - Review. Saudi J. Biol. Sci., v. 20, p.105-120, 2013.

CHUNG, C.; DEGNER, B.; McCLEMENTS, D.J. Understanding multicomponent emulsionbased products: Influence of locust bean gum on fat droplet - Starch granule mixtures. Food Hydrocoll., v. 35, p. 315-323, 2014.

CIRIGLIANO, M.C.; CARMAN, G.M. Purification and characterization of liposan, a bioemulsifier from Candida lipolytica. Appl. Environ. Microbiol., v. 50, p. 846-850, 1985.

COOPER, D. G.; CAVALERO, D. A. The effect of medium composition on the structure and physical state of sophorolipids produced by Candida bombicola ATCC 22214. J. Biotechnol., v. 103, p. 31-41, 2003.

HEYMAN, B.; DE VOS, W.H.; DEPYPERE, F.; VAN DER MEEREN, P.; DEWETTINCK, K. Guar and xanthan gum differentially affect shear induced breakdown of native waxy maize starch. Food Hydrocoll., v. 35, p. 546-556, 2014.

KRALOVA, I.; SJÖBLOM, J. Surfactants Used in Food Industry: A Review. J. Dispers. Sci. Technol., v. 30, p.1363-1383, 2009.

LI, J.; WANG, Y.; JIN, W.; ZHOU, B.; LI, B. Application of micronized konjac gel for fat analogue in mayonnaise. Food Hydrocoll., v. 35, p.375-382, 2014.

MARCHANT, R.; BANAT, I.M. Microbial biosurfactants: challenges and opportunities for future exploitation. Trends Biotechnol., v. 11, p. 558-565, 2012. 
MARINESCU, G.; STOICESCU, A.; PATRASCU, L. The preparation of mayonnaise containing spent brewer's yeast $\beta$-glucan as a fat replacer. Romanian Biotechnol. Lett., v. 16, p. 60176025, 2011.

MUTHUSAMY, K.; GOPALAKRISHNAN, S.; RAVI, T,K.; SIVACHIDAMBARAM, P. Biosurfactants: properties, commercial production and application. Curr. Sci., v. 94, p. 736747, 2008.

NADARAJAH, D.; HAN, J.H.; HOLLEY, R.A. Use of mustard flour to inactivate Escherichia coli $\mathrm{O}$ 157:H7 in ground beef under nitrogen flushed packaging. Int. J. Food Microbiol., v. 99, p. 257-267, 2005.

NITSCHKE, M.M.; COSTA S.G.V.O. Biosurfactant in food industry. Trends in Food Sci. Technol., v. 18, p. 252-259, 2007.

PACWA-PLOCINICZAK, M.; PLAZA, G.A.; PIOTROWSKA-SEGET, S.; CAMEOTRA, S.S. Environmental Applications of biosurfactants: recent advances. Int. J. Mol. Sci., v. 12, p.633-654, 2011.

PARASKEVOPOULOU, D.; BOSKOU, D.; PARASKEVOPOULOU, A. Oxidative stability of olive oil-lemon juice salad dressings stabilized with polysaccharides. Food Chem., v. 101, p. 1197-1204, 2007.

SATO, A.C.K.; MORAES, K.E.F.P.; CUNHA, R.L. Development of gelled emulsions with improved oxidative and pH stability. Food Hydrocoll., v. 34, p. 184-192, 2014.

TORABIZADEH, H.; SHOJAOSADATI, S.A.; TEHRANI H.A. Preparation and characterization of bioemulsfier from Saccharomyces cerevisiae and its application in food products. Lebensmittel-Wissenschaft \& Technologie, v. 29, p. 734-737, 1996.

TSAO, R.; YU, Q.; FRIESEN, I.; POTTER, J.; CHIBA, M.. Factors affecting the dissolution and degradation of oriental mustard-derived sinigrin and allyl isothiocyanate in aqueous media. $J$. Agric. Food Chem., v. 48, p. 1898-1902, 2000. 